\title{
Association of MUC2, MUC5AC and MUC5B genes with the recurrence of nasal polyps
}

\author{
LEI LIU, CHAOHUI YAN and SHUDONG TAO
}

\begin{abstract}
Department of Otorhinolaryngology and Head and Neck Surgery, The Third Central Hospital of Tianjin, Tianjin Key Laboratory of Extracorporeal Life Support for Critical Diseases, Artificial Cell Engineering Technology Research Centre, Tianjin Institute of Hepatobiliary Disease, Tianjin 300170, P.R. China
\end{abstract}

Received October 27, 2019; Accepted April 29, 2020

DOI: $10.3892 /$ etm.2020.8837

\begin{abstract}
Although mucins were suggested to contribute to the pathogenesis of nasal polyposis, the correlation between the expression levels of MUC5AC, MUC5B and MUC2, and the recurrence of nasal polyps, has not been extensively investigated. The present study aimed to investigate the association of the levels of mucin (MUC) 2, MUC5AC and MUC5B with the recurrence of nasal polyps. A total of 56 patients with nasal polyps who underwent functional endoscopic sinus surgery at the Tianjin Third Central Hospital (Tianjin, China) between June 2007 and June 2010 were included and baseline characteristics were recorded. Reverse transcription-quantitative PCR analysis was used to determine the expression levels of the MUC2, MUC5AC and MUC5B genes at six months following the operation. The recurrence rate was calculated at one year following the operation. Spearman's rank correlation was used to determine the association between the reduction in the expression levels of MUC2, MUC5AC and MUC5B, and the recurrence rate of nasal polyps. There were no significant differences observed in the baseline characteristics between patients with and without the recurrence of nasal polyps. Prior to treatment, the expression levels of MUC5AC, MUC5B and MUC2 in patients with nasal polyps were significantly increased compared with those in the paranasal tissues and normal nasal mucosa. The expression levels of MUC5AC, MUC5B and MUC2 were similar between patients with and without recurrent nasal polyps. In addition, significantly decreased MUC5AC, MUC5B and MUC2 gene expression levels were observed in patients without recurrence of nasal
\end{abstract}

Correspondence to: Dr Chaohui Yan, Department of Otorhinolaryngology and Head and Neck Surgery, The Third Central Hospital of Tianjin, Tianjin Key Laboratory of Extracorporeal Life Support for Critical Diseases, Artificial Cell Engineering Technology Research Centre, Tianjin Institute of Hepatobiliary Disease, 83 Jintang Road, Hedong, Tianjin 300170, P.R. China

E-mail: ychtjszx@126.com

Key words: nasal polyps, mucin 5AC, mucin 5B, mucin 2, recurrence polyps compared with those with recurrence of nasal polyps at six months following the operation. The decreased values of MUC5AC, MUC5B and MUC2 in patients with recurrence and without recurrence of nasal polyps compared with baselines were significantly negatively correlated with the recurrence rate of nasal polyps. In conclusion, the present results provided novel data for the diagnosis and treatment of patients with recurrent nasal polyps.

\section{Introduction}

As a subgroup of chronic rhinosinusitis, nasal polyposis is a clinical manifestation with a complex pathogenesis, which involves chronic local infection, a family history and genetic predisposition, atopy and allergy, as well as aerodynamic factors (1). Although nasal polyps are only detected in a small percentage of adults, they have been discovered to lead to headaches, facial pain, fatigue, hyposmia or total loss of the sense of smell and taste (2). In children, the occurrence of nasal polyps has been associated with cystic fibrosis or cilia dysfunction (3). Unfortunately, the subjective nature of this disease makes it difficult to quantify successfully, and patients with nasal polyps usually fail to be fully cured due to the complex mechanisms underlying the pathogenesis and recurrence (2).

Mucins (MUC) are mainly synthesized by goblet cells and submucosal glandular cells in the airways (4). The MUC5AC, MUC5B and MUC2 genes, which have all been proven to serve a pivotal role in inflammatory respiratory diseases $(5,6)$, are expressed in the sinus and mixed nasal mucus secretions of patients with nasal polyps (7). It was previously demonstrated that downregulation of MUC1 was associated with corticosteroid resistance in patients with nasal polyps (8). In addition, MUC5AC and MUC5B were discovered to contribute to the pathogenesis of nasal polyposis and the development of chronic rhinosinusitis $(9,10)$. It has also been suggested that elevated levels of MUC2 and MUC5B may induce excessive mucus secretion and decreased mucociliary clearance in patients with chronic rhinosinusitis (11). However, to the best of our knowledge, the correlation between the expression levels of MUC5AC, MUC5B and MUC2 and the recurrence of nasal polyps has not been extensively investigated. 
Therefore, the present study aimed to investigate the expression levels of MUC2, MUC5AC and MUC5B in patients with and without the recurrence of nasal polyps, in addition to determining whether MUC expression levels were associated with recurrence.

\section{Materials and methods}

Patients. A total of 56 patients with nasal polyps who underwent functional endoscopic sinus surgery (FESS) at The Third Central Hospital of Tianjin (Tianjin, China) between June 2007 and June 2010 were included in the present study. The inclusion criteria were as follows: Male or female patients $>18$ years old and patients with suspected nasal polyps were treated surgically and nasal polyps were confirmed by pathology. Post-operative pathology confirmed that all of the patients had nasal polyps. Among the 56 patients, 39 were males and 17 were females, and the median age was 45.5 years with a range of 18-66 years. A total of 12 patients ( 7 males and 5 females, aged 17-56 years with a median age of 40.5 years) without nasal polyps underwent nasal septal reconstruction. Patients with a history of food allergy, drug allergy, smoking, allergic rhinitis, asthma, polypectomy, or oral or nasal spray hormone use within two months prior to surgery were excluded. All tissue samples were stored at $-80^{\circ} \mathrm{C}$. The study protocol was approved by the Ethics Committee of The Third Central Hospital of Tianjin (Tianjin, China) and informed consent was obtained verbally from all patients.

Experimental procedure. Pre-operative routine medical history, pre-operative nasal endoscopy, sinus CT examination and scoring were performed using Lund-Mackay CT score (12). The pre-operative endoscopic examination revealed the presence of new translucent grape-like organisms in the nasal cavity. Considering the presence of nasal polyps, the location and scope of the polyps were determined by an experienced otolaryngologist, who identified polypoid via nasal endoscopy before operation, and a Lund-Kennedy endpoint score (13) was given. Polypectomy and open sinus surgery were performed according to the principles of FESS (14). During the operation, nasal polyp tissues from the middle nasal meatus and nasal mucosal tissues without polyps (paranasal tissues) were collected to determine the expression levels of MUC2, MUC5AC and MUC5B. After the surgery, patients were administered external treatment $(64 \mu \mathrm{g}$ budesonide nasal spray, once in each nostril, twice in the morning and evening, continuous application for $\geq 12$ weeks) and daily nasal flushing with saline solution for 6 months. The subjects were also subjected to regular endoscopy examination (every 2 weeks for 6 months) and surgical cavity cleaning (every 4 weeks for 6 months), including dry scabs and vesicles, to check for recurrent polyps. At 6 months after surgery, the majority of the recurrent polyps were located in the operative cavity of the open ethmoid sinus or partially open ethmoid sinus in the middle nasal meatus, rather than completely filling the middle nasal meatus. However, there were also other mucosal tissues besides the polyps. Thus, the epithelialized nasal mucosal tissues were collected again to determine the expression levels of MUC2, MUC5AC and MUC5B. At 1 year post-surgery, 43 patients were recurrence-free, whereas 13 patients demonstrated recurrence for nasal polyps. In addition, 12 normal mucosal tissue samples were collected following nasal septal reconstruction, and MUC2, MUC5AC and MUC5B expression levels were also detected. The patients without colds were re-examined using nasal endoscopy after the operation. If gray litchi flesh-like tissues were observed in the middle nasal tract, the patients were given budesonide nasal spray treatment for 2 weeks. After 2 weeks of nasal hormone therapy, a pathological biopsy was taken if lychee-like tissues were still present. Pathology confirmed that these tissues were polyp tissues, which was subsequently defined as the recurrence of nasal polyps.

Extraction and quantitative analysis of total RNA. The total RNA Isolation kit (Promega Corp.) was used according to the manufacturer's protocol to extract total RNA from tissue. Together with $1 \mathrm{ml}$ RNA lysate and $7 \mu \mathrm{l} \beta$-mercaptoethanol (Sigma-Aldrich; Merck KGaA), $0.1 \mathrm{~g}$ tissue was fully ground and collected in Eppendorf tubes (Eppendorf). NaAC ( $2 \mathrm{~mol} / \mathrm{l}$; $\mathrm{pH}$ 4.0; Sigma-Aldrich; Merck KGaA), $1 \mathrm{ml}$ water-saturated phenol and $200 \mathrm{ml}$ chloroform/isoamyl alcohol (49:1; Sigma-Aldrich; Merck KGaA) were added in succession. The mixture was fully homogenized by violent oscillation and placed on ice for $15 \mathrm{~min}$. Following centrifugation at $4^{\circ} \mathrm{C}$ for $15 \mathrm{~min}$ at $12,000 \mathrm{x} \mathrm{g}$, the supernatant was carefully collected and an equal volume of isopropanol (Thermo Fisher Scientific, Inc.; 99.5\%; HPLC grade) was added following precipitation at $-20^{\circ} \mathrm{C}$ for $>1 \mathrm{~h}$. Subsequently, $300 \mu \mathrm{l}$ pancreatic RNase A (Sigma-Aldrich; Merck KGaA) was added and re-precipitation was performed by adding an equal volume of isopropanol. After washing with precooled $75 \%$ ethanol (Sigma-Aldrich; Merck $\mathrm{KGaA}$ ) for $10 \mathrm{sec}$, the precipitate was dissolved in $100 \mathrm{ml}$ diethyl pyrocarbonate (DEPC)-treated water (Sigma-Aldrich; Merck KGaA). The RNA concentration was measured using a spectrophotometer (NanoDrop Technologies; Thermo Fisher Scientific, Inc.).

Reverse transcription-quantitative ( $q P C R)$. First-strand complementary (c)DNA was synthesized in a total volume of $20 \mu \mathrm{l}$ containing $1 \mu \mathrm{g}$ total RNA, $6.2 \mu \mathrm{l}$ random primer pd(N) (Roche Diagnostics), DEPC-treated water, $4 \mu 1$ 5X RT buffer (Invitrogen; Thermo Fisher Scientific, Inc.), $1 \mu \mathrm{l}$ avian myeloblastosis virus reverse transcriptase (Roche Diagnostics $\mathrm{GmbH}), 2.4 \mu 1 \mathrm{MgCl}_{2}$ (25 mM; Sangon Biotech Co., Ltd.) and $1 \mu \mathrm{l}$ deoxynucleoside triphosphates $(10 \mathrm{mM})$. The temperature protocol was $25^{\circ} \mathrm{C}$ for $15 \mathrm{~min}, 42^{\circ} \mathrm{C}$ for $80 \mathrm{~min}$ and $70^{\circ} \mathrm{C}$ for $10 \mathrm{~min}$. qPCR was subsequently performed in a total volume of $24 \mu \mathrm{l}$, containing $2 \mu \mathrm{l}$ template, $20 \mu \mathrm{l}$ Real-Time PCR Master Mix (SYBR Green; Roche Diagnostics), $0.5 \mu \mathrm{l} 10 \mu \mathrm{M}$ primer (Table I) and $1 \mu \mathrm{l}$ Taq DNA polymerase (U/ $\mu \mathrm{l}$; AmpliTaq; PerkinElmer, Inc.). The following thermocycling conditions were used for the qPCR: Initial denaturation at $95^{\circ} \mathrm{C}$ for $2 \mathrm{~min}$; followed by 40 cycles at $94^{\circ} \mathrm{C}$ for $60 \mathrm{sec}$ and $60^{\circ} \mathrm{C}$ for $45 \mathrm{sec}$. The quantification cycle $(\mathrm{Ct})$ values were obtained according to the real-time amplification curve and melting curve of each gene. The relative expression levels of the target genes were calculated using the $2^{-\Delta \Delta \mathrm{Cq}}$ method (15).

Immunohistochemical analysis. The normal nasal mucosa in the control group and the nasal polyp tissues were fixed in 
Table I. Primers used for the reverse transcription-quantitative PCR.

\begin{tabular}{|c|c|c|c|}
\hline Gene & Position (nucleotides) & Primer sequences & Size of product (bp) \\
\hline \multirow[t]{2}{*}{ G3PDH } & $915-935 \mathrm{nt}$ & Forward 5'-GGGCATCCTGGGCTACACTGA-3' & \\
\hline & $1057-1032 \mathrm{nt}$ & Reverse 5'-CAAATTCGTTGTCATACCAGGAAATG-3' & 143 \\
\hline \multirow[t]{2}{*}{ MUC2 } & $12884-12901 \mathrm{nt}$ & Forward 5'-AACCCACACCGCCCCTGC-3' & \\
\hline & $13256-13237 \mathrm{nt}$ & Reverse 5'ACGGCCCCGTTAAGCACAGC-3' & 373 \\
\hline \multirow[t]{2}{*}{ MUC5AC } & $2743-2763 \mathrm{nt}$ & Forward 5'-ATGGAGGCTGCTGAGGGACAG-3' & \\
\hline & $3258-3239 \mathrm{nt}$ & Reverse 5'GTGGCCGTTGACCAGGACGG-3' & 516 \\
\hline \multirow[t]{2}{*}{ MUC5B } & $15475-15494 \mathrm{nt}$ & Forward 5'-GCCGACAGCAGCTTCACCGT-3' & \\
\hline & $15617-15598 \mathrm{nt}$ & Reverse 5'TTGAGGAACACGCCGCCGTC-3' & 143 \\
\hline
\end{tabular}

MUC, mucin.

$4 \%$ neutral formalin for $24 \mathrm{~h}$, embedded in paraffin and cut into 5- $\mu \mathrm{m}$ sections for immunohistochemical staining. The sections were deparaffinized using Histoplast PE Paraffin at $65^{\circ} \mathrm{C}$ and rehydrated using anhydrous ethanol for $5 \mathrm{~min}$, $95 \%$ ethanol for $5 \mathrm{~min}, 80 \%$ ethanol for $5 \mathrm{~min}$ and $75 \%$ ethanol for $5 \mathrm{~min}$, and then subjected to antigen retrieval using EDTA (pH 9.0). Deparaffinized sections were incubated with $3 \% \mathrm{H}_{2} \mathrm{O}_{2}$ (Sangon Biotech Co., Ltd.) for $10 \mathrm{~min}$ at $37^{\circ} \mathrm{C}$ to inhibit endogenous peroxidase activity and subsequently incubated with goat serum (Gibco; Thermo Fisher Scientific, Inc.) to block non-specific binding sites. The tissue sections were incubated with anti-mouse MUC5AC (1:200; Abcam; cat. no. ab3649), MUC5B (1:250; Abcam; cat. no. ab87376) and MUC2 (1:200; Abcam; cat. no. ab134119) monoclonal primary antibodies at $4^{\circ} \mathrm{C}$ for $10 \mathrm{~min}$. Following the incubation with primary antibody, the sections were washed with PBS for 2 min and incubated for $30 \mathrm{~min}$ with a secondary antibody (Polyperoxidase-anti-mouse/rabbit IgG; 1:200; cat. no, JHA01; Shanghai Jiehao Biotechnology Co., Ltd) at $37^{\circ} \mathrm{C}$. The slides were subsequently stained with $3,3^{\prime}$-diaminobenzidine at $37^{\circ} \mathrm{C}$ for $30 \mathrm{~min}$, followed by counterstaining with hematoxylin at $37^{\circ} \mathrm{C}$ for $1 \mathrm{~min}$, dehydration using $75 \%$ ethanol for $5 \mathrm{~min}$, $80 \%$ ethanol for $5 \mathrm{~min}, 95 \%$ ethanol for $5 \mathrm{~min}$ and anhydrous ethanol for $5 \mathrm{~min}$ ) and mounting by neutral gum seal. The yellowish to brownish in the cell membrane or cytoplasm was considered to indicate positive cells. Five high power fields (OLYMPUS-BX53; Olympus Corporation; magnification, $\mathrm{x} 400$ ) were randomly selected for analysis. To evaluate staining, a score of (a) the percentage of positive cells in each field of view (0, no positive cells; $1,1-29 \%$ positive cells; $2,30-59 \%$ positive cells; $3, \geq 60 \%$ positive cells) $*(b)$ the staining intensity (0, non-staining; 1 , faint yellow; 2 , brown yellow; 3 , sepia) was considered to be a comprehensive score: $<2$ was negative and $\geq 2$ was positive. The results of the staining were assessed by two independent pathologists in a blinded manner.

Statistical analysis. All experiments were repeated in triplicate. Statistical analysis was performed using SPSS version 16.0 software (SPSS, Inc.). The experimental data are presented as the median and interquartile range. In Table II, the categorical variable sex was analyzed using a $\chi^{2}$-test. Differences between two groups were determined using a Mann-Whitney U-test. Spearman's rank correlation was used for correlation analysis. $\mathrm{P}<0.05$ was considered to indicate a statistically significant difference.

\section{Results}

Patient characteristics. A total of 56 patients with nasal polyps who underwent FESS were included in the present study. The included patients were divided into the recurrence group $(n=13)$ and the non-recurrence group $(n=43)$. No significant differences were observed in the mean age, sex, previous nasal polyp, Lund-Mackay CT score, Lund-Kennedy endoscopy score, hospital stay, duration of the operation, post-operative re-examinations or the post-operative use of nasal sprays between patients with recurrence and those without recurrence (P>0.05; Table II).

Expression levels of MUC5AC, MUC5B and MUC2 prior to treatment. The expression levels of MUC5AC, MUC5B and MUC2 in the nasal polyp tissues prior to treatment were significantly upregulated compared with those in the paranasal tissues and normal nasal mucosal tissues $(\mathrm{P}<0.01$; Fig. $1 \mathrm{~A}-\mathrm{C})$. However, no significant differences were identified in the expression levels of MUC5AC, MUC5B and MUC2 mRNA between patients with recurrent nasal polyps and those without recurrent nasal polyps prior to treatment ( $\mathrm{P}>0.05$; Fig. 1D-F).

Immunohistochemical analysis of MUC5AC, MUC5B and $M U C 2$ expression levels. To determine the localization and expression levels of MUC5AC, MUC5B and MUC2, immunohistochemical staining was performed prior to treatment. Positive MUC5AC, MUC5B and MUC2 staining was mainly localized in the membrane and cytoplasm of epithelial and goblet cells, and it was less prominent in the glandular cells (Fig. 2A). Compared with the normal nasal mucosal tissues, the levels of MUC5AC $(\mathrm{P}=0.02)$, MUC5B $(\mathrm{P}=0.05)$ and MUC2 $(\mathrm{P}=0.01)$ were significantly elevated in the nasal polyps (Fig. 2B).

Expression levels of MUC5AC, MUC5B and MUC2 following treatment. Among the 56 patients who underwent FESS and drug treatment, 43 were observed to have complete epithelialization of the nasal mucosa and no recurrence of nasal polyps was observed one year later; however, 13 patients presented 
Table II. Association between the recurrence rate and clinicopathological features of patients with nasal polyps.

\begin{tabular}{lcrc}
\hline Variable & Total (n=56) & $\begin{array}{c}\text { Patients with } \\
\text { recurrence (n=13) }\end{array}$ & $\begin{array}{c}\text { Patients without } \\
\text { recurrence (n=43) }\end{array}$ \\
\hline Age (years) & $45.51 \pm 11.9$ & $45.82 \pm 11.9$ & $44.15 \pm 12.11$ \\
Sex & & & 0.78 \\
Male & $39(69.6)$ & $9(69.2)$ & 0.97 \\
Female & $17(30.4)$ & $4(30.8)$ & $30(69.8)$ \\
Previous nasal polyp (months) & $5.65 \pm 1.62$ & $6.67 \pm 1.15$ & $13(30.2)$ \\
Lund-Mackay CT score & $21.20 \pm 2.54$ & $21.71 \pm 2.45$ & $4.51 \pm 1.24$ \\
Lund-Kennedy endoscopy score & $11.48 \pm 0.81$ & $11.84 \pm 0.98$ & $20.81 \pm 2.51$ \\
Hospital stay (days) & $5.92 \pm 0.51$ & $5.54 \pm 0.91$ & $0.92 \pm 0.73$ \\
Duration of operation (h) & $1.55 \pm 0.35$ & $1.67 \pm 0.23$ & $6.01 \pm 0.82$ \\
Post-operative re-examinations (n) & $7.81 \pm 0.51$ & $8.02 \pm 0.92$ & 0.55 \\
Post-operative use of nasal spray hormone (weeks) & $13.02 \pm 0.41$ & $14.24 \pm 0.62$ & 0.71 \\
\hline
\end{tabular}

Values are expressed as $\mathrm{n}(\%)$ or mean \pm standard deviation.
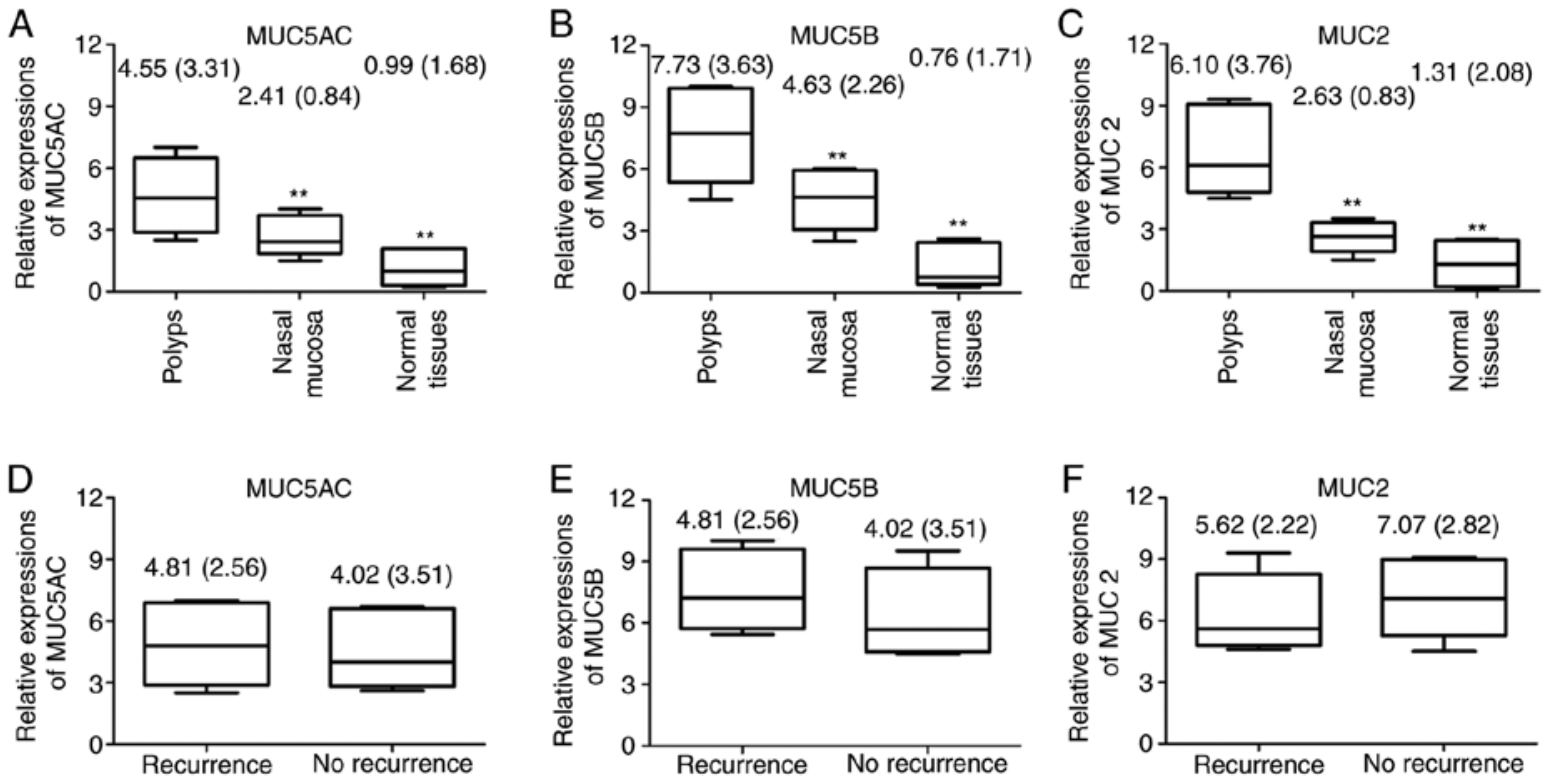

Figure 1. Relative expression levels of MUC5AC, MUC5B and MUC2 prior to treatment. Relative expression levels of (A) MUC5AC, (B) MUC5B and (C) MUC2 in the nasal polyps, nasal mucosa and normal tissues prior to treatment were analyzed using RT-qPCR. Relative expression levels of (D) MUC5AC, (E) MUC5B and (F) MUC2 in patients with and without the recurrence of nasal polyps at six months after operation were analyzed using RT-qPCR. Data are shown as median (interquartile). ${ }^{* *} \mathrm{P}<0.01$ vs. nasal polyp tissues. MUC, mucin; RT-qPCR, reverse transcription-quantitative PCR.

with recurrent nasal polyps. Patients without recurrence of nasal polyps were discovered to have significantly decreased expression levels of MUC5AC, MUC5B and MUC2 compared with those in patients with recurrence of nasal polyps at 6 months after the operation $(\mathrm{P}<0.01$; Fig. 3A-C). Furthermore, a greater reduction (at 6 months compared with the baseline) of MUC5AC, MUC5B and MUC2 expression levels was observed in patients without nasal polyp recurrence compared with that in patients with nasal polyp recurrence $(\mathrm{P}<0.01$; Fig. 3D-F).

Correlation analysis of MUC5AC, MUC5B and MUC2 expression levels with the recurrence rate of nasal polyps. Correlation analysis of the reduction in the expression levels of MUC5AC, MUC5B and MUC2 with the recurrence rate of nasal polyps at one year after the operation was then performed. The decreased values of MUC5AC $\left(\mathrm{r}^{2}=0.83\right.$; $\mathrm{P}<0.01)$, MUC5B $\left(\mathrm{r}^{2}=0.68 ; \mathrm{P}<0.01\right)$ and MUC2 $\left(\mathrm{r}^{2}=0.65\right.$; $\mathrm{P}<0.01)$ in patients with recurrence and without recurrence of nasal polyps at six months compared with baseline levels were revealed to be significantly negatively correlated with the recurrence rate of nasal polyps (Fig. 4A-C).

\section{Discussion}

Submucosal glandular cells and superficial epithelial goblet cells produce various MUC proteins (9). Excessive secretion of 

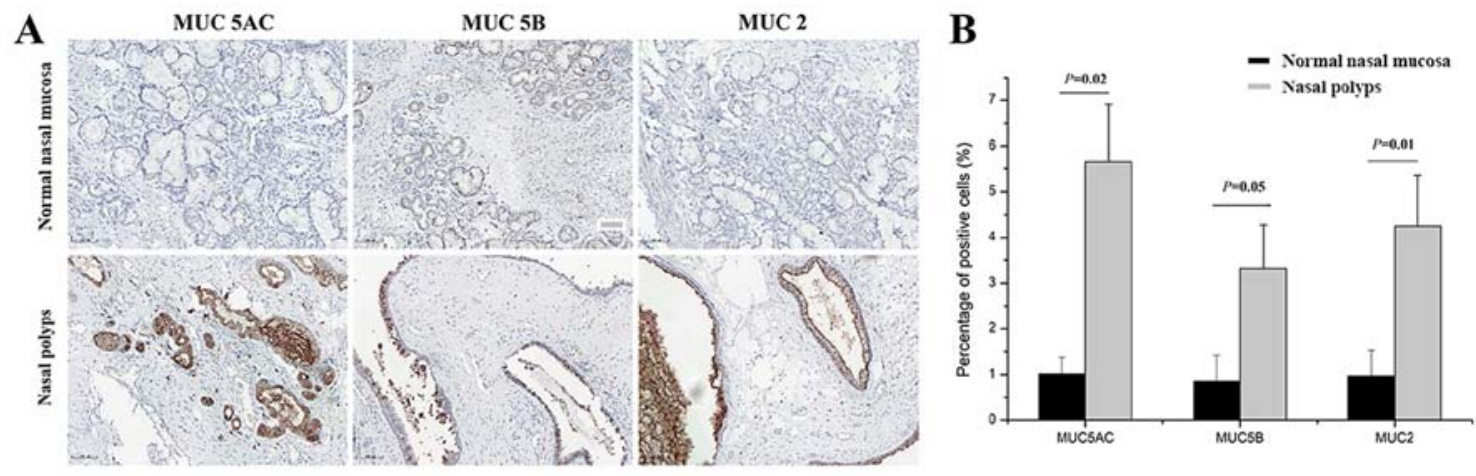

Figure 2. Immunohistochemical analysis of MUC5AC, MUC5B and MUC2 expression levels in normal nasal mucosa and nasal polyps. (A) Immunohistochemical staining for MUC5AC, MUC5B and MUC2 expression levels in normal nasal mucosa tissues and nasal polyps (magnification, x400). (B) Semi-quantitative analysis of the expression levels of MUC5AC, MUC5B and MUC2 in normal nasal mucosa tissues and nasal polyps. MUC, mucin.
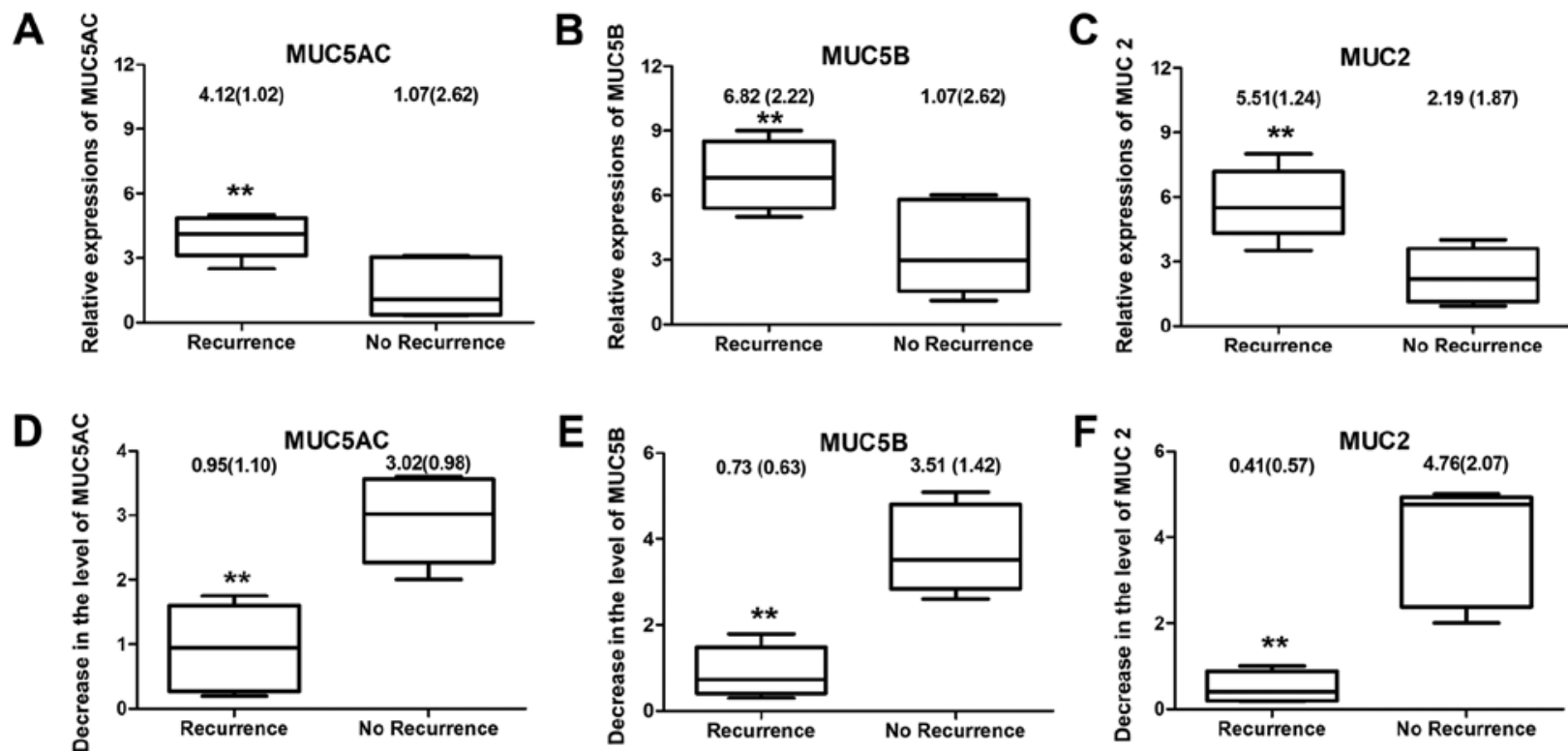

Figure 3. Relative expression levels of MUC5AC, MUC5B and MUC2 following treatment. Relative expression levels of (A) MUC5AC, (B) MUC5B and (C) MUC2 in patients with and without recurrence of nasal polyps following treatment were analyzed using reverse transcription-quantitative PCR. The decrease in the levels of (D) MUC5AC, (E) MUC5B and (F) MUC2 in patients with and without the recurrence of nasal polyps at six months after operation vs. baselines were calculated. Data are shown as median (interquartile). ${ }^{* *} \mathrm{P}<0.01$ vs. no recurrence. MUC, mucin.
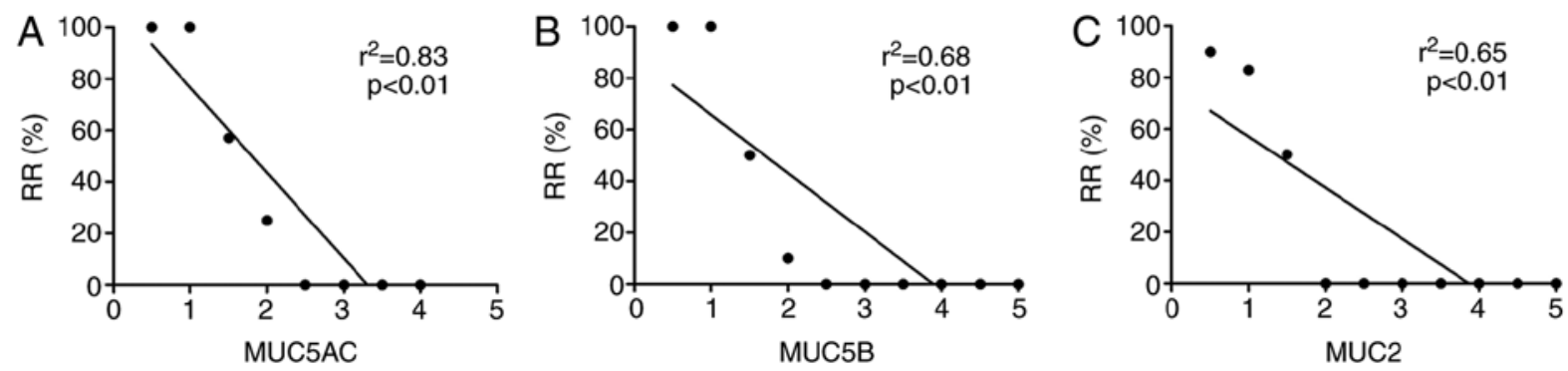

Figure 4. Correlation analysis between MUC5AC, MUC5B and MUC2 expression levels with the recurrence rate of nasal polyps. Correlation analysis was performed to determine the correlation between (A) MUC5AC, (B) MUC5B and (C) MUC2 and the recurrence rate of nasal polyps. RR, recurrence rate; MUC, mucin.

mucus was identified to be one of the major symptoms of nasal polyposis, which may be the result of the increased quantity of mucus and/or changes in its physical properties (16). Studies on human MUC genes, including MUC2, MUC5AC and
MUC5B, have been previously performed (17,18); however, to the best of our knowledge, there are no detailed studies on the role of MUC5AC, MUC5B and MUC2 in patients presenting with nasal polyp recurrence, and their association with the 
recurrence of nasal polyps has yet to be fully elucidated. To investigate the association of MUC5AC, MUC5B and MUC2 with the recurrence of nasal polyps, the expression levels of MUC5AC, MUC5B and MUC2 were detected in patients with and without nasal polyp recurrence. a correlation analysis was also performed to determine whether these MUC genes were correlated with the recurrence rate of nasal polyps. The results of the present study revealed that the expression levels of MUC5AC, MUC5B and MUC2 in nasal polyp tissues prior to treatment were significantly increased. However, following FESS and medical treatment, significantly decreased expression levels of MUC5AC, MUC5B and MUC2 were observed in patients without recurrence compared with those with recurrent nasal polyps. Correlation analysis also demonstrated that the decrease in the expression levels of MUC5AC, MUC5B and MUC2 was significantly negatively correlated with the recurrence rate of nasal polyps.

In a previous study, proteins in the sinonasal secretions of pediatric patients were analyzed by mass spectrometry, revealing that MUC5B and MUC5AC were present in the majority of the samples, and the relative abundance of MUC5B was indicated to be significantly higher in pediatric patients with chronic rhinosinusitis (CRS) (19). Kim et al (20) evaluated the expression levels of MUC5AC and MUC5B in 20 chronic rhinosinusitis samples and discovered that the expression levels of MUC5AC and MUC5B mRNA were markedly increased compared with those in the normal sinus mucosa. In addition, Poachanukoon et al (21) demonstrated that the expression levels of MUC2 and MUC5AC genes, as well as tumor necrosis factor (TNF)- $\alpha$ production, were inhibited following treatment with mometasone fuorate (MF) and budesonide (BUD), indicating that MF and BUD may attenuate the pathogenic effects of MUC2 and MUC5AC in phorbol-12-myristate-13-acetate-induced human airway epithelial cells. The results of the present study were consistent with these previous studies; in the present study, increased expression levels of MUC2, MUC5AC and MUC5B were identified in patients with recurrent nasal polyps.

In a study on 24 patients with CRS, Mao et al (10) assessed the expression status of MUC1, MUC2, MUC5AC and MUC5B on the bacterial biofilm (BBF; + or -status) in patients with CRS. It was discovered that patients in the BBF (+) CRS group had significantly increased expression levels of MUC5AC and MUC5B in the sinus mucosa compared with those in the BBF (-) CRS group, whereas no significant differences were observed in the expression levels of MUC1 and MUC2 between the two groups. Contrary to the results, the present study included 56 patients with nasal polyps who underwent FESS, and it was discovered that the expression levels of the MUC2 gene were significantly increased in the patients with nasal polyp recurrence compared with those in patients without recurrence. These different outcomes may be explained by the differences in patients included in the two studies.

In CRS, upregulation of MUC5AC and MUC5B was determined in the sinus mucosa of BBF in CRS, suggesting that the elevated expression of MUC5AC and MUC5B may serve an important role in the pathogenesis of BBF formation (10). Infection of mucosal epithelial cells by Shigella species was also indicated to lead to intense and acute inflammatory bowel disease, and elevated expression levels of MUC2 and MUC5AC within 6-8 h via activating TNF- $\alpha$, protein kinase $\mathrm{C}$ and ERK1/2 (22). Tos and Mogensen (23) proposed the theory of epithelial rupture during the formation of nasal polyps, in which the infiltration and edema in the nasal mucosa resulted in the rupture of the epithelium and the formation of granulations, which gradually become lined with pseudostratified columnar epithelium. Following the epithelial rupture, mucosal herniation, re-epithelialization, new gland formation and finally, the formation of nasal polyps was observed. Thus, in the present study, MUC genes were investigated to determine their association with the recurrence of nasal polyps. The results suggested that upregulated expression levels of MUC2, MUC5AC and MUC5B were observed in patients with nasal polyps and that the reduction in MUC5AC, MUC5B or MUC2 expression levels was significantly negatively correlated with the recurrence rate of nasal polyps. These results indicated that the inflammation associated with nasal polyps may lead to the increased expression levels of MUC5AC, MUC5B and MUC2.

Following treatment, the results of the current study demonstrated that increased expression levels of MUC genes remained; this is most likely due to the incomplete resistance to current treatment or incomplete removal of nasal polyps following an operation, the epithelization of nasal mucosa or incomplete restoration of surface ciliary function, leading to the retention of mucus secretions and bacteria, and the persistence of various inflammatory factors. This would contribute to the high expression levels of MUC genes and eventually nasal polyp recurrence. Therefore, if the expression levels of MUC genes are not reduced, research should focus on actively determining the causes in order to intervene as early as possible to avoid the recurrence of polyps.

Although the expression levels of MUC5AC, MUC5B and MUC2 were proven to be associated with the recurrence rate of nasal polyps, there were certain limitations to the present study. First, the present study had a relatively small sample size, which may introduce selection bias. In addition, it was a single-center study and lacks external validation. Thus, further multicenter and large-sample studies are required to validate the present results.

In conclusion, the present study demonstrated that the expression levels of MUC5AC, MUC5B and MUC2 were significantly negatively correlated with the recurrence rate of nasal polyps. These results may provide novel evidence for the diagnosis and treatment of patients with recurrent nasal polyps.

\section{Acknowledgements}

Not applicable.

\section{Funding}

No funding was received.

\section{Availability of data and materials}

The datasets generated and/or analyzed during the current study are available from the repository of Tianjin Third Central Hospital (Tianjin, China) http://www.tj3zx.cn/. The datasets 
used and/or analyzed during the current study are available from the corresponding author on reasonable request.

\section{Authors' contributions}

LL performed the experiments, collected the data and wrote the manuscript; CHY designed the experiments and revised the manuscript; and SDT analyzed the data and revised the manuscript. All authors read and approved the final manuscript.

\section{Ethics approval and consent to participate}

The study protocol was approved by the Ethics Committee of The Third Central Hospital of Tianjin (Tianjin, China) and informed consent was obtained verbally from all patients. The analysis was retrospective.

\section{Patient consent for publication}

Not applicable.

\section{Competing interests}

The authors declare that they have no competing interests.

\section{References}

1. Pawliczak R, Lewandowska-Polak A and Kowalski ML: Pathogenesis of nasal polyps: An update. Curr Allergy Asthma Rep 5: 463-471, 2005

2. Deal RT and Kountakis SE: Significance of nasal polyps in chronic rhinosinusitis: Symptoms and surgical outcomes. Laryngoscope 114: 1932-1935, 2004.

3. Bequignon E, Dupuy L, Zerah-Lancner F, Bassinet L, Honoré I, Legendre M, Devars du Mayne M, Escabasse V, Crestani B Maître B, et al: Critical evaluation of sinonasal disease in 64 adults with primary ciliary dyskinesia. J Clin Med 8: 619, 2019.

4. Crystal RG, Randell SH, Engelhardt JF, Voynow J and Sunday ME: Airway epithelial cells: Current concepts and challenges. Proc Am Thorac Soc 5: 772-777, 2008.

5. Perrais M, Pigny P, Copin MC, Aubert JP and Van Seuningen I: Induction of MUC2 and MUC5AC mucins by factors of the epidermal growth factor (EGF) family is mediated by EGF receptor/Ras/Raf/extracellular signal-regulated kinase cascade and Sp1. J Biol Chem 277: 32258-32267, 2002.
6. Henke MO, John G, Germann M, Lindemann $\mathrm{H}$ and Rubin BK: MUC5AC and MUC5B mucins increase in cystic fibrosis airway secretions during pulmonary exacerbation. Am J Respir Crit Care Med 175: 816-821, 2007.

7. Ali MS, Wilson J and Pearson JP: Mixed nasal mucus as a model for sinus mucin gene expression studies. Laryngoscope 112: 326-331, 2002.

8. Milara J, Peiró T, Armengot M, Frias S, Morell A, Serrano A and Cortijio J: Mucin 1 downregulation associates with corticosteroid resistance in chronic rhinosinusitis with nasal polyps. J Allergy Clin Immunol 135: 470-476, 2015.

9. Ding GQ and Zheng CQ: The expression of MUC5AC and MUC5B mucin genes in the mucosa of chronic rhinosinusitis and nasal polyposis. Am J Rhinol 21: 359-366, 2007.

10. Mao YJ, Chen HH, Wang B, Liu X and Xiong GY: Increased expression of MUC5AC and MUC5B promoting bacterial biofilm formation in chronic rhinosinusitis patients. Auris Nasus Larynx 42: 294-298, 2015.

11. Xue-Kun H, Yuan L, Jin Y, Peng L and Hong L: Expression of MUC2 and MUC5B in ethmoid sinus mucosa of patients with chronic rhinosinusitis. Sci Res Essays 5: 1690-1696, 2010.

12. Lund VJ and Kennedy DW: Staging for rhinosinusitis. Otolaryngol Head Neck Surg 117 (Suppl): S35-S40, 1997.

13. Lund WJ and Kennedy DW: Quantification for staging. The Staging and Therapy Group. Ann Otol Rhinol Laryngol 167 (Suppl): S17-S21, 1995.

14. Kennedy DW: Functional endoscopic sinus surgery. Technique. Arch Otolaryngol 111: 643-649, 1985

15. Livak KJ and Schmittgen TD: Analysis of relative gene expression data using real-time quantitative PCR and the 2-(-Delta Delta C(T)) method. Methods 25: 402-408, 2001.

16. Ali MS, Wilson JA, Bennett M and Pearson JP: Mucin gene expression in nasal polyps. Acta Otolaryngol 125: 618-624, 2005

17. Chang JH, Song KJ, Kim HJ, Kim JH, Kim NH and Kim KS: Dietary polyphenols affect MUC5AC expression and ciliary movement in respiratory cells and nasal mucosa. Am J Rhinol Allergy 24: e59-e 62, 2010.

18. İlhan Ö, Han Ü, Önal B and Celık SY: Prognostic significance of MUC1, MUC2 and MUC5AC expressions in gastric carcinoma. Turk J Gastroenterol 21: 345-352, 2010.

19. Saieg A, Brown KJ, Pena MT, Rose MC and Preciado D: Proteomic analysis of pediatric sinonasal secretions shows increased MUC5B mucin in CRS. Pediatr Res 77: 356-362, 2015.

20. Kim DH, Chu HS, Lee JY, Hwang SJ, Lee SH and Lee HM: Up-regulation of MUC5AC and MUC5B mucin genes in chronic rhinosinusitis. Otolaryngol Head Neck Surg 130: 747-752, 2004.

21. Poachanukoon O, Koontongkaew S, Monthanapisut P and Pattanacharoenchai N: Mometasone furoate suppresses PMA-Induced MUC-5AC and MUC-2 production in human airway epithelial cells. Tuberc Respir Dis (Seoul) 80: 60-68, 2017.

22. Radhakrishnan P, Halagowder D and Devaraj SN: Altered expression of MUC2 and MUC5AC in response to Shigella infection, an in vivo study. Biochim Biophys Acta 1770: 884-889, 2007.

23. Tos $\mathbf{M}$ and Mogensen C: Pathogenesis of nasal polyps. Rhinology 15: 87-95, 1977. 